\title{
Light at the end of the tunnel?
}

Tim O'Brien

Associate Professor/Honorary Consultant in Restorative Dentistry, Peninsula Dental School and Expert witness

The BDJ Upfront section includes editorials, letters, news, book reviews and interviews. Please direct your correspondence to the News Editor,

Kate Quinlan at BDJNews@nature.com. Press releases or articles may be edited, and should include a colour photograph if possible.

\section{L} ike many, I was pleased by the publication by the General Dental Council (GDC) of its proposals for a better and fairer system of regulation. ${ }^{1}$ To me, this document is a welcome move towards recognition that avoidable harm of patients needs to be seen within the context of the wider system, rather than purely a problem caused by a 'bad apple. This approach is very much in keeping with others involved in the issue of patient safety, and demonstrates the first step towards dealing with a regulatory system that has arguably lost its focus in recent years.

Such an approach is not without challenges; one of the biggest is the lamentable lack of published data to underpin such a policy, particularly in relation to what is known as 'avoidable' harm. We are currently in a situation where nobody truly knows where and when avoidable harm happens to patients within primary care dentistry. Consequently, our understanding of why it is happening is, in many cases, based on conjecture. It is only by understanding these issues that we can put effective systems in place to better protect our patients. As my wise old tutors from dental school would have said 'You can't form a treatment plan without an examination and diagnosis'.

Shifting the balance sensibly proposes that educators should have data shared with them that show how their teaching may influence patient care, but it is just as important that others involved in healthcare systems also receive useful information. Bodies involved in contracting could gain useful data about which areas of their activity hold the greatest risk and formulate strategies to quality assure appropriately. I would hope that commissioners are carefully considering recent cases where systematic failures, involving thousands of patients, were identified, and learn relevant lessons from it.

Not only are there few data available but, when they do exist, they are often held by bodies who often sit squarely on opposite sides of the fence. So, while it is laudable that the GDC has decided to shift its focus in this way, this surely needs to be met with an appropriate response from the profession. Cases that reach the stage of civil or regulatory proceedings are only a small proportion of instances of harm, and so it is just as important to learn from 'near misses' and lesser levels of harm. Learning from reporting systems and civil proceedings should also be used to inform regulatory strategy but, if we are to use these data, we will need to try to rebuild trust between the parties involved.
The move towards local resolution, where appropriate, is to be commended, but there is equally a need to ensure that there is funding to support this and that judgments are made in the fair and proportionate manner way that the current Fitness to Practise panels generally do. A 'postcode lottery' must be avoided at all costs, and decisions must be adequately defensible to avoid appeals negating the value of the process.

Reassuringly, there is recognition from both sides of the fence that a change in approach is needed. I also support this but, bearing in mind the missed opportunities

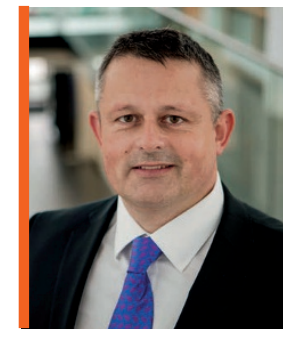

\section{'...nobody truly knows where and when avoidable harm happens to patients within primary care dentistry...'}

There is a need to ensure that 'proportionate' regulation is adequately supported. We need to be sure that the issues that trigger assessments of 'Fitness to Practise' are genuinely those that hold a threat to patients, and avoid regulation simply becoming a 'tick-box' exercise that misses the important issues. Again, this area lacks data and our understanding of the issues that cause the most harm to patients is rudimentary. Arguably, performance assessments of any type should be informed by data from actual cases of avoidable harm, rather than what one or two experts think may or may not be important. The General Medical Council has published its view on when a matter is likely to suggest that Fitness to Practise is impaired, and it could be argued that a similar standard could be used for dentistry. Their comments on 'isolated' cases are of particular interest ${ }^{2}$ and this seems to me to be a sensible place to start if the process is to be proportionate. in recent years to learn lessons from other industries, including our colleagues in medicine, I would humbly suggest that this should not be left exclusively to 'lay people'; it requires engagement from the wider profession if it is to be effective.

I am genuinely more hopeful now than ever that changes can be made which will not only better protect patients but, as importantly, will not hinder the 'reasonable' practitioner from carrying out their daily task of helping patients to improve their health. It's just a shame that it has taken so long for this to come about.

DOI: 10.1038/sj.bdj.2017.690

1. GDC. Shifting the balance: a better, fairer system of dental regulation. March 2017. Available at https:// www.gdc-uk.org/about/what-we-do/regulatory-reform (Accessed 3 August 2017).

2. GMC. The meaning of Fitness to Practise. March 2013 Available at http://www.gmc-uk.org/guidance/21721. asp (Accessed 3 August 2017). 